\title{
Full Order Observer Based Control of Single-phase Inverter Both in Standalone and Gridtie Modes
}

\author{
Nagulapati Kiran \\ Anil Neerukonda Institute of Technology and Management, Sangivalasa, Visakhapatnam, Andhra \\ Pradesh, India \\ ${ }^{*}$ Corresponding author, e-mail: nkiran.ped@gmail.com
}

\begin{abstract}
In 3-phase systems, the reliability of 3-phase Inverter is not good. In such situations Paralleled Inverter Systems are used. Parallel Inverter operation has a major topic in uninterruptible power system (UPS) applications where the design is focused on the standalone operation, and output stage is typically an inductor-capacitor filter. Most of the standalone inverter systems use a LC filter and proportional-integral (PI) controller in their control loops. When connecting the paralleled inverters to utility grids, the capacitor becomes redundant and thus either a pure inductor or an LCL filter can be used as inverter output stage. Compared with the L filter, the LCL filter is more attractive because it cannot only provide higher harmonics attenuation with same inductance value, but also allow inverter to operate both in standalone and grid-tie modes, which makes it a universal inverter for distributed generation applications. Output of Inverter should always be checked. In this paper, a full order observer for Inverter System is designed and is used for estimation of State Variables of the Inverter System. Two modes are adopted: a) Grid-tie Mode b) Standalone Mode. MATLAB/Simulink is used to verify and validate the proposed idea.
\end{abstract}

Keywords: single phase inverter, standalone mode, grid tie mode, state space model, LCL filter, full order oserver

\section{Introduction}

Inverters are predominantly used as interface link to process and convert it to the form suitable for utility because of the control flexibility they provide. Inverters convert power from dc to ac with controllable voltage and variable frequency. For battery interface applications, inverters are used as a bidirectional link to charge batteries during surplus periods and to discharge them in absence of power. Hence inverters take power from different modules and deliver it to common grid, which is synchronized with local utility grid or in islanded mode [1-2].

This requires the parallel operation of inverters. Paralleled Inverters can be used in place of one large single unit. The parallel operation of small inverters gives modularity to the system and provides extra reliability and redundancy [3]. Increase in the number of inverters increases the stiffness of the system and makes it more stable against external disturbances. Hence the parallel operation of inverters offers several advantages in addition to low maintenance cost of lower power unit compared to that of high power unit [4-5].

The stability of large-scale distributed generation systems was analyzed by state space model. This model is relatively easy to expand by combining single inverter state space equations to build state space equations for paralleled inverter systems [6-8].

In this paper, the state space model is adopted to investigate the stability of the paralleled inverter system. The state space model of single inverter in both standalone and grid-tie mode will be obtained. This can be extended to $n$ number of inverters connected in parallel. Here the observer estimates state variables and the difference between the estimated state variables and the actual state variables must tend to zero, which in other words means that the impulse response of overall system must be zero. MATLAB/SIMULINK is used to verify the model.

\section{State Observers}

In the design of control systems, we assume that all state variables are available for feedback. In practice, however not all state variables are available for feedback. Then we need to estimate unavailable state variables. Estimation of immeasurable state variables is commonly 
called observation. A device (or a computer program) that estimates or observes the state variables is called an observer. If the state observer observes all state variables of the system, regardless of whether some state variables are available for direct measurement, it is called full order state observer. There are times when this will not be necessary when we need observation of only the immeasurable state variables but not of those that are directly measurable. An observer that estimates fewer than $n$ state variables, where $n$ is the dimension of state vector, is called a reduced order state observer or simply a reduced order observer. If the order of reduced order state observer is minimum possible, the observer is called a minimum order state observer or minimum order observer.

\subsection{Full Order State Observer}

A state observer estimates the state variables based on the measurements of the output and control variables. Concept of Observability and Controllability plays an important role. The observer is a subsystem to reconstruct the state vector of the plant. The mathematical model of the observer is basically the same as that of the plant, except that we include an additional term that includes the estimation error to compensate for inaccuracies in matrices $A$ and $B$ and the lack of initial error. The estimation error or observation error is the difference between the measured output and the estimated output. The initial error is the difference between the initial state and initial estimated state. Thus the mathematical model of the observer is defined to be,

$$
\hat{x}=A x+B u+K e(Y-C x)
$$

It can be re-written as

$$
\hat{x}=A-K e C x+B u+K e Y
$$

Where is estimated state and $\mathrm{C} x$ is the estimated output. The inputs to the observer are the output $\mathrm{Y}$ and the control input $\mathrm{u}$. Matrix $\mathrm{Ke}$ which is observer gain matrix, is a weighting matrix to the corrected term involving the difference between the measured output $Y$ and estimated output $\mathrm{C} x$. This term continuously corrects the model output and improves the performance of the observer. Figure 1 shows the block diagram of the system and full-order observer.

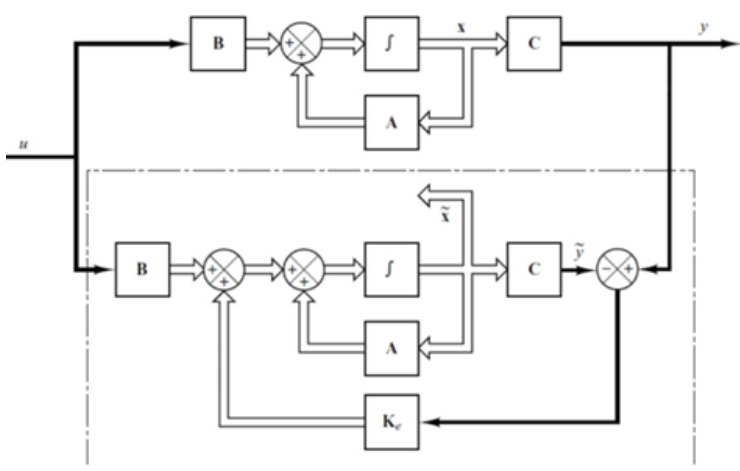

Figure.1 Block diagram of system and full order state observer when input $\mathrm{u}$ and output y are scalars

Both the system and the full order observer have common input $u$. The difference between actual and estimated state is defined as error vector.

$$
X-\hat{x}=A-\operatorname{Ke}(x-\hat{x})
$$

If the matrix is a stable matrix, the error vector will converge to zero for any initial vector. It means $x(t)$ will converge to $\mathrm{x}(\mathrm{t})$ regardless of the values of $\mathrm{x}(0)$ and $x(0)$. If the eigen values 
of the matrix $(\mathrm{A}-\mathrm{KeC})$ are chosen in such a way that the dynamic behavior of the error vector will tend to zero with an adequate speed.

If the plant is completely observable, then it can be proved that it is possible to choose matrix $K e$ such that $(A-K e C)$ has arbitrarily desired eigen values.

\subsection{Transfer Function of Observer based Controller}

$$
\begin{aligned}
& \dot{x}=A x+B u \\
& y=C x
\end{aligned}
$$

Assume that the plant is completely observable and use observed-state feedback control

$$
u=-K \widetilde{x}
$$

Then the equation for the observer are given by

$$
\widehat{x}=\left(A-K_{e} C-B K\right) \hat{x}+K_{e} y
$$

By taking Laplace transform of Equation (3.15), assuming a zero initial condition and solving we obtain:

$$
\hat{X}(s)=\left(s I-A+K_{e} C+B K\right)^{-1} K_{e} Y(s)
$$

Thereby

$$
\widehat{U}(s)=-K\left(s I-A+K_{e} C+B K\right)^{-1} K_{e} Y(s)
$$

Hense the transfer function can be obtained as

$$
\frac{U(s)}{Y(s)}=-K\left(s I-A+K_{e} C+B K\right)^{-1} K_{e}
$$

We can notice that the transfer function obtained acts as a controller for the system

$$
K\left(s I-A+K_{e} C+B K\right)^{-1} K_{e}
$$

This transfer function is called observer based controller transfer. Figure 2 shows the block diagram representation for the system.

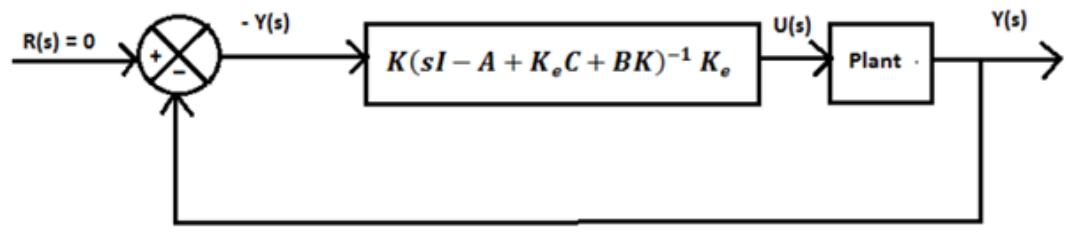

Figure 2. Block diagram representation of system with a controller-observer 


\section{State Space Modelling}

\subsection{Standalone Mode}

The circuit configuration of Single Inverter in Standalone mode is shown in Figure 3. A LCL filter is commonly used for filtering the output current ripple in standalone mode. Three state variables iac, iload and vc are inverter side inductor current, load current and capacitor voltage respectively. $d$ is the excitation signal.

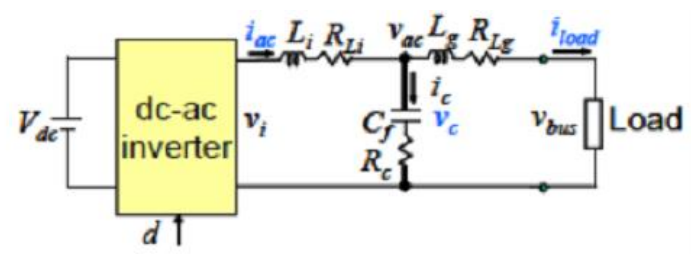

Figure 3. Block Diagram of Single Shase Inverter in Standalone Mode

If the load is only a pure resistor with resistance Rload, then the plant equation can be expressed by a third-order system.

$$
\left[\begin{array}{ccc}
L_{i} & 0 & R_{f} C_{f} \\
0 & L_{g} & R_{f} C_{f} \\
0_{i} & 0 & C_{f}
\end{array}\right]\left[\begin{array}{c}
\frac{d i_{a c}}{d t} \\
\frac{d i_{\text {load }}}{d t} \\
\frac{d i a c}{d v_{c}} \\
\frac{d t}{d t}
\end{array}\right]=\left[\begin{array}{ccc}
R_{L 1} & 0 & -1 \\
0 & -R_{L g}-R_{\text {load }} & 1 \\
1 & -1 & 0
\end{array}\right]\left[\begin{array}{c}
i_{a c} \\
i_{\text {load }} \\
V_{c}
\end{array}\right]+\left[\begin{array}{c}
V_{d c} \\
0 \\
0
\end{array}\right][d]
$$
assuming:

The state equation of Single Inverter model in Standalone mode can be obtained by

$$
\mathrm{Lg}=40 \mathrm{mH}, \mathrm{Li}=50 \mathrm{mH}, \mathrm{Cf}=0.02 \mathrm{~F}, \mathrm{Rc}=10 \Omega, \mathrm{RLg}=6 \Omega, \mathrm{RLi}=5 \Omega, \mathrm{RLoad}=13.5 \Omega
$$

$$
\begin{aligned}
& {\left[\begin{array}{c}
\dot{i}_{\text {ac }} \\
\dot{i}_{\text {load }} \\
\dot{v}_{c}
\end{array}\right]=\left[\begin{array}{ccc}
100 & 0 & -1050 \\
0 & 487.5 & -482.5 \\
20 & -25 & -450
\end{array}\right]\left[\begin{array}{c}
i_{a c} \\
i_{\text {load }} \\
v_{c}
\end{array}\right]+\left[\begin{array}{c}
8700 \\
0 \\
0
\end{array}\right][d]} \\
& Y=\left[\begin{array}{lll}
1 & 0 & 0
\end{array}\right]\left[\begin{array}{c}
i_{\text {ac }} \\
i_{\text {load }} \\
V_{c}
\end{array}\right]
\end{aligned}
$$

where,

$$
A=\left[\begin{array}{ccc}
100 & 0 & -1050 \\
0 & 487.5 & -482.5 \\
20 & -25 & -450
\end{array}\right] B=\left[\begin{array}{c}
8700 \\
0 \\
0
\end{array}\right] C=\left[\begin{array}{lll}
0 & 0 & 1
\end{array}\right]
$$

\subsection{Grid-tie Mode}

Here the inverter is feeding an L-C filter. State variables $\mathrm{iL}$ and vc are inverter side inductor current, and capacitor voltage respectively. VE is the excitation source. Figure 4 shows circuit configuration of single phase inverter in standalone mode. Figure 5 shows closed loop simulink model of transfer function model of inverter without full order observer (standalone 
mode). Figure 6 shows closed loop simulink model of transfer function model of inverter with full order observer (standalone mode). Figure 7 shows closed loop simulink model of transfer function model inverter without full order observer. Figure 8 shows closed loop simulink model of transfer function model of inverter with full order observer.

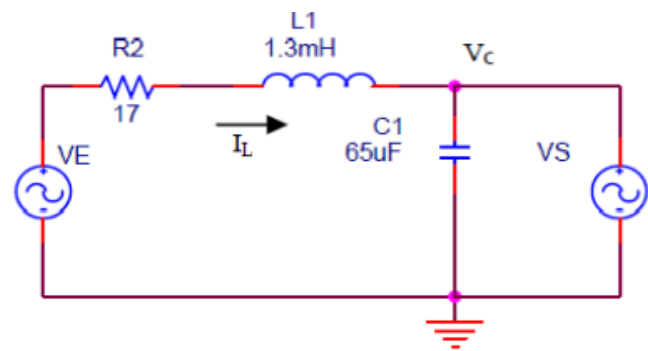

Figure 4. Circuit configuration of single phase inverter in standalone mode

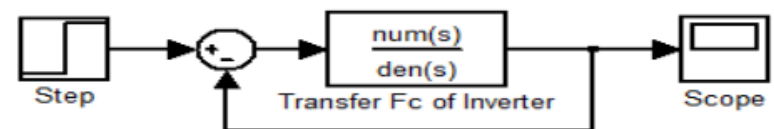

Figure 5. Closed loop simulink model of transfer function model of inverter without full order observer (standalone mode)

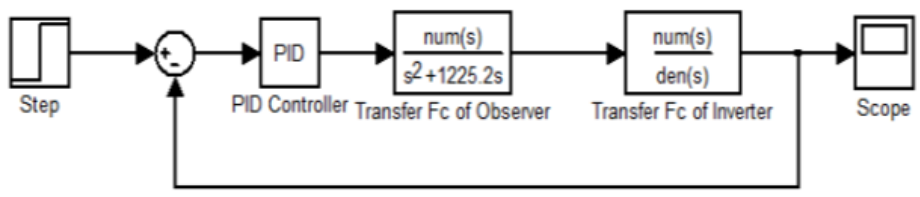

Figure 6. Closed loop simulink model of transfer function model of inverter with full order observer (standalone mode)

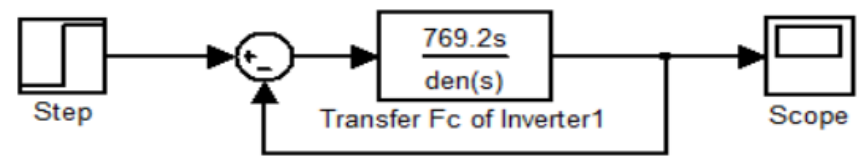

Figure 7. Closed loop simulink model of transfer function model inverter without full order observer

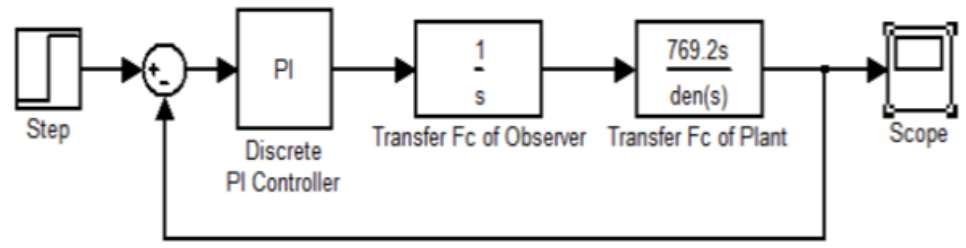

Figure 8. Closed loop simulink model of transfer function model of inverter with full order observer

The General State equation of Single phase Inverter in Grid-tie mode can be expressed as: 


$$
\left[\begin{array}{l}
\frac{d V_{c}}{d t} \\
\frac{d_{i L}}{d t}
\end{array}\right]=\left[\begin{array}{cc}
0 & \frac{1}{C} \\
\frac{-1}{L} & \frac{-R}{L}
\end{array}\right]\left[\begin{array}{l}
V_{c} \\
i_{L}
\end{array}\right]+\left[\begin{array}{l}
0 \\
\frac{1}{L}
\end{array}\right]\left[V_{E}\right]
$$
assuming:

The state equation of Single Inverter model in Grid-tie mode can be obtained by $L=1.3 \mathrm{mH}, \mathrm{R}=1 \Omega, \mathrm{C}=65 \mu \mathrm{F}$

$$
\begin{aligned}
& {\left[\begin{array}{l}
\frac{d V_{c}}{d t} \\
\frac{d_{i L}}{d t}
\end{array}\right]=\left[\begin{array}{cc}
0 & 15380 \\
-769.2 & -769.2
\end{array}\right]\left[\begin{array}{l}
V_{c} \\
i_{L}
\end{array}\right]+\left[\begin{array}{c}
0 \\
769.2
\end{array}\right]\left[V_{E}\right]} \\
& Y=\left[\begin{array}{ll}
0 & 1
\end{array}\right]\left[\begin{array}{l}
V_{c} \\
i_{L}
\end{array}\right]
\end{aligned}
$$

where,

$$
A=\left[\begin{array}{rrrr}
0 & & 15380 & \\
-769 & .2 & -769 & .2
\end{array}\right] \quad B=\left[\begin{array}{cc}
0 & \\
769 & .2
\end{array}\right] \quad C=\left[\begin{array}{ll}
0 & 1
\end{array}\right]
$$

\section{Transfer Functions}

The obtained transfer functions of inverter and full order observer in both standalone and grid-tie mode are shown below:

\subsection{Standalone Mode}

\subsubsection{Transfer Function of Inverter}

$$
\frac{174000 s+84825000}{s^{3}+837.5 s^{2}+26000 s+362500}
$$

\subsubsection{Transfer Function of Full order observer}

$$
\frac{s^{2}+3.51 s-447.8}{s^{2}+1225.2 s}
$$

4.2. Grid-tie Mode

4.2.1. Transfer Function of Inverter

$$
\frac{769.2 s}{s^{2}+769.2 s+11830000}
$$

\subsubsection{Transfer Function of Full Order Observer}

$$
\frac{1}{s}
$$


5. Simulink Models

5.1. Standalone Mode:

5.1.1. Closed Loop without Full order Observer

Unit Step is given as input to the transfer function model of Inverter. Its unit step response is given in Figure 9.

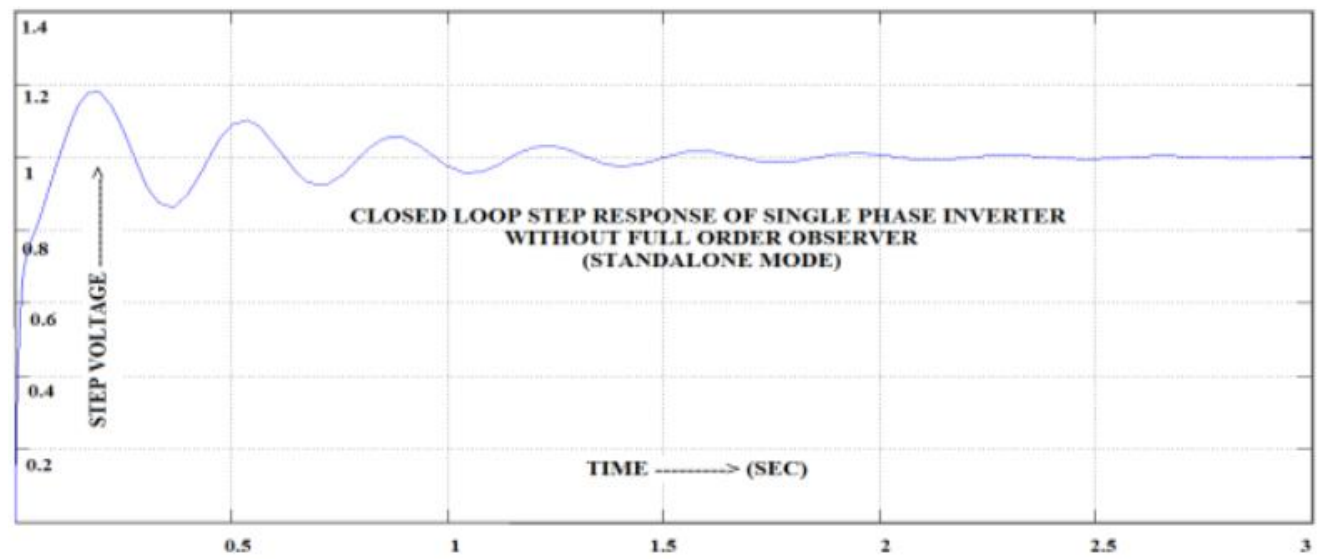

Figure 9. Waveform of closed loop unit step response of single-phase inverter without full order observer (standalone mode)

\subsubsection{Closed Loop with Full Order Observer}

Unit Step is given as input to the PI Controller. Its output is given as input to the transfer function model of Full order Observer. Its output is given as input to the transfer function model of Inverter. Its step response is given in Figure 10.

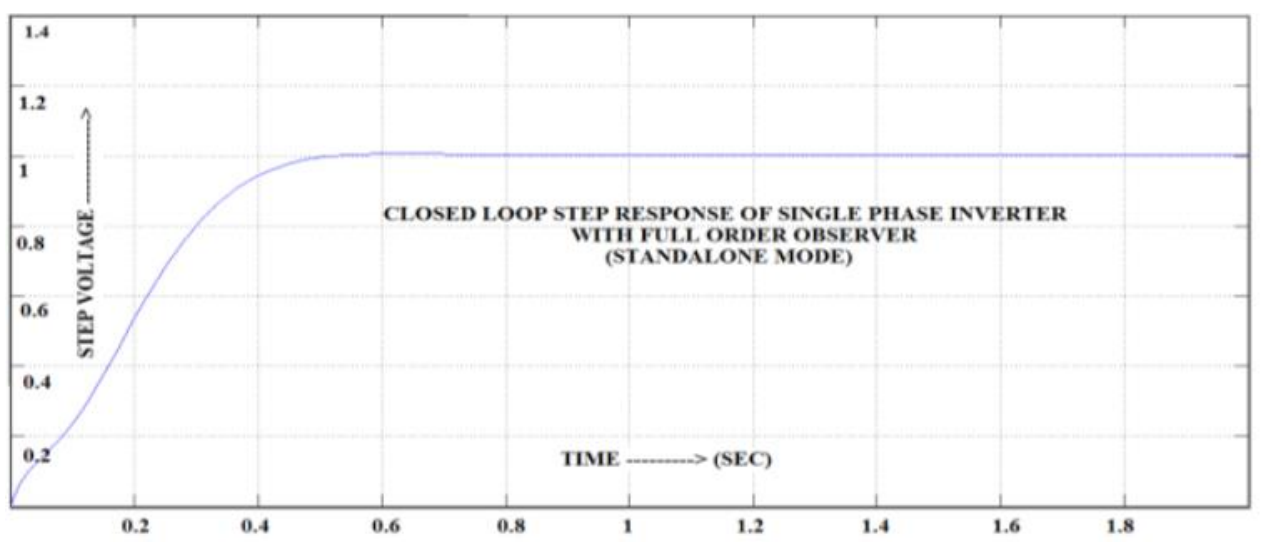

Figure 10. Waveform of closed loop unit step response of single-phase inverter with full order observer (standalone mode)

\subsection{Grid Tie Mode}

\subsubsection{Closed Loop without Full order Observer}

Unit Step is given as input to the transfer function model of Inverter. Its unit step response is given in Figure 11. 


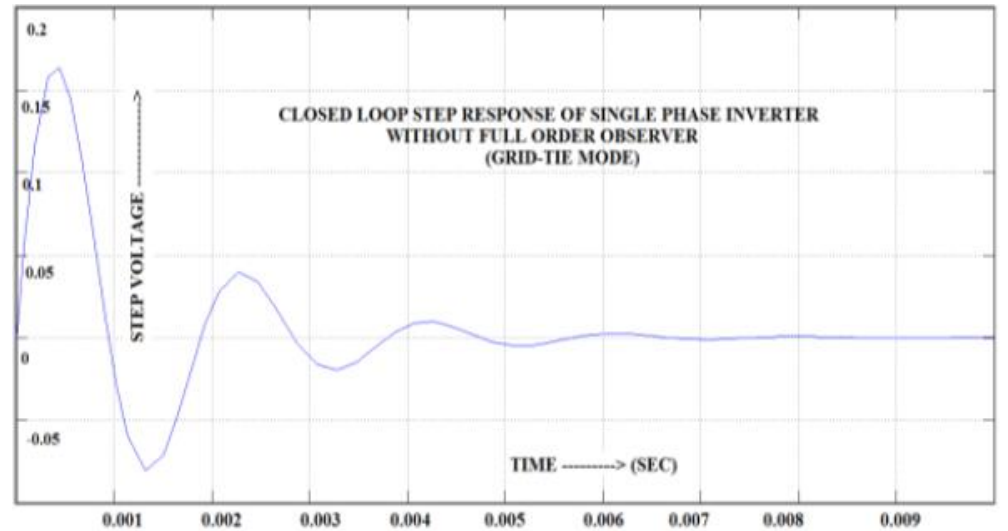

Figure 11. Waveform of closed loop unit step response of single phase inverter without full order observer (grid tie mode)

\subsubsection{Closed Loop with Full Order Observer}

Unit Step is given as input to the PI Controller. Its output is given as input to the transfer function model of Full Order Observer. Its output is given as input to the transfer function model of Inverter. Its step response is given in Figure 12.

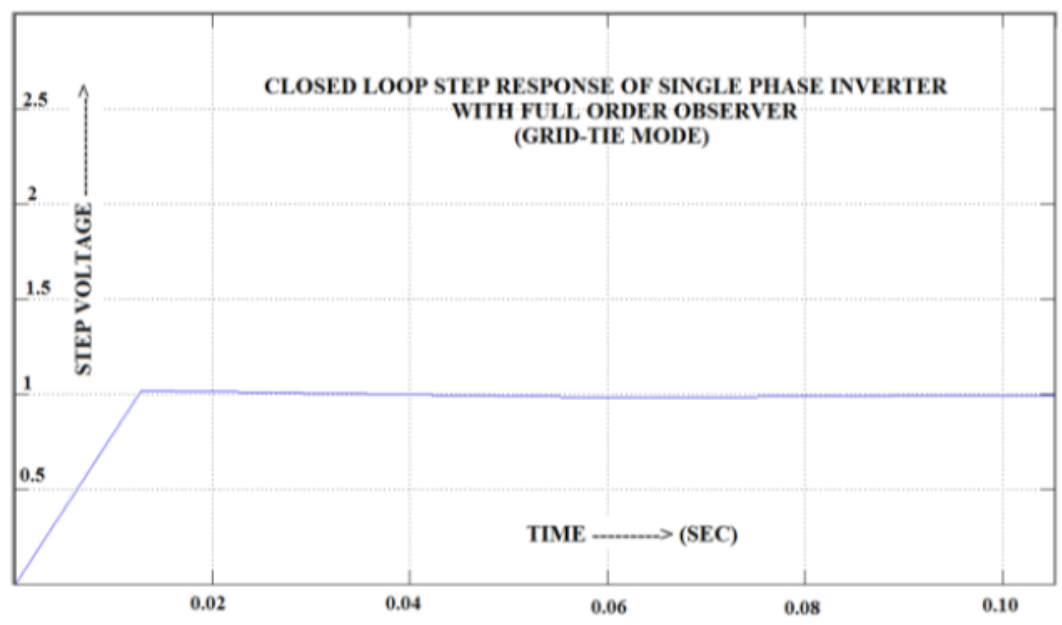

Figure 12. Waveform of open loop unit step response of single phase inverter with full order observer

\section{Simulation Results}

6.1. Standalone Mode

\subsubsection{Closed Loop Unit Step Response without Observer}

Closed Loop Unit Step Response of Single Phase Inverter without Full Order Observer is shown in Figure 9.

Since the unit step response has reached steady state at unit step voltage, the system considered is stable. Even though the system is stable, we can observe that without Full Order Observer there many oscillations in the obtained response.

\subsubsection{Closed Loop Unit Step Response with Observer}

Closed Loop Unit Step Response of Single Phase Inverter with Full Order Observer is shown in Figure 10. It can be observed that the waveform is reaching steady state around 0.5 
seconds. Since the unit step response is reaching steady state at unit step voltage, the system considered is stable. We can observe that with Full Order Observer, there are no more oscillations in the response, hence the unit step response of such a system has improved.

\subsection{Grid Tie Mode}

\subsubsection{Closed Loop Unit Step Response without Observer}

Unit Step response for Transfer function model of inverter without Full order observer in grid tie mode is shown in Figure 11. It can be observed that without Full Order Observer the response is tending to zero and has many oscillations. It is not the desired step response.

\subsubsection{Closed Loop Unit Step Response with Observer}

Unit Step response for Transfer function model of inverter with full order Observer in Gridtie mode is shown in Figure 12. It can be observed that the waveform is reaching steady state value around 0.01 seconds. Since the unit step response is reaching steady state at unit step voltage, the system considered is stable. We can observe that with Full Order Observer, there are no more oscillations in the response, hence the unit step response of such a system has improved.

\section{Conclusion}

In this paper, two modes have been adopted;

1) Standalone mode 2) Grid-tie mode Transfer function of single phase inverter and Full order Observer in both Standalone and Grid-tie mode is obtained. Using State Space modeling, the state variables have been estimated and State Equations have been obtained for single unit 3-phase Inverter in both the modes.

Using MATLAB/SIMULINK, both Open loop and closed loop system have been simulated in both the modes. Unit Step response of the OLS without Full order Observer, OLS with Full order Observer, CLS without Full order Observer, CLS with Full order Observer, were obtained and were compared in both modes.

\section{References}

[1] Chien Liang Chen, Jih-Sheng Lai, Martin D. State-space modeling, analysis and implementation of paralleled inverters for micro grid applications. Applied Power electronics Conferences and Exposition (APEC). Twenty-Fifth Annual IEEE. 2010.

[2] Talebi N, Sadrina, MA, Rafiei, SRM. Current and Voltage control Paralleled Multi module inverter systems. Control and Automatation. MED'09. 17th Mediterranean Conference. 2009.

[3] Xunbo Fu, Chunliang E, Jianlin Li. Modeling and simulation of parallel-operation grid connected inverter. Industrial Technology. ICIT 2008. IEEE Conference. 2008.

[4] Chien-Liang Chen, Jih-Sheng Lai, Yu-Bin Wang, Sung-Yeul Park Miwa, H. Design and Control for LCLBased Inverters with Both Grid-Tie and Standalone Parallel Operations. Industry Applications Society Annual Meeting 2008, IAS '08. IEEE. 2008.

[5] Hoff, E, Skjellnes, T Norum, L. Paralleled three-phase inverters. Department of Electrical power Engineering, Norwegian University of Science and technology, NTNU.

[6] Katusukhiko Ogata. Modern Control Engineering. University of Minnesota.

[7] A Nagoor Kani. Advanced Control Theory.

[8] Sun, YS Lee, DH Xu, Modeling, analysis, and implementation of parallel multi-inverter systems with instantaneous Average-current-sharing scheme. IEEE Trans. Power Electron. 2003; 18: 844-856. 